\title{
Jürgen Grieser: Elternarbeit in der Psychotherapie von Kindern und Jugendlichen Göttingen: Vandenhoeck und Ruprecht, 2018
}

\author{
Norbert Wolff(Wetzikon)
}

Die Arbeit mit den Eltern stelle in der Psychotherapie von Kindern und Jugendlichen eine oft unterschätzte Herausforderung dar, schreiben Inge SeiffgeKrenke und Franz Resch im Vorwort zum Band von Jürgen Grieser in der Reihe «Psychodynamik kompakt». Zudem wird die Elternarbeit auch im wissenschaftlichen Diskurs der Fachliteratur vernachlässigt. Dabei stelle sie gemäss Grieser «den eigentlichen Knackpunkt und die wahre Herausforderung in der therapeutischen Arbeit dar, sie kann für das Gelingen oder Misslingen des ganzen therapeutischen Prozesses entscheidend sein» (S. 11). So sehen die Psychotherapierichtlinien in der BRD Elternarbeit im Verhältnis 1:4 vor. Als Ziel der Elternarbeit definiert Grieser die «Wiederentdeckung der Freude am Kind» (vgl. das Buch von Datler et al., 1999).

Im ersten Kapitel legt der Autor dar, Warum es ohne die Eltern nicht geht-in erster Linie, weil das Kind von den Eltern abhängig ist und diese es in die Therapie bringen. Die häufigeren Behandlungsabbrüche in Psychotherapien mit Kindern sind oftmals auf den ungenügenden Einbezug der Eltern zurückzuführen. Diese müssen die Kränkung bewältigen können, eine Therapie für ihr Kind zu benötigen, und sie müssen in der Lage sein, Veränderungen im familiären System und bei sich selbst zuzulassen und zu vollziehen. In einer kurzen Darstellung der Geschichte der Elternarbeit geht Grieser auf die verschiedenen Perspektiven und Haltungen der Pionierinnen der Kinderanalyse Anna Freud, Melanie Klein, D. Burlingham und H. Hug Hellmuth ein. Inzwischen wird die begleitende Arbeit mit den Eltern nicht mehr nur als notwendiges Übel, sondern als zentraler Bestandteil der therapeutischen Arbeit mit Kindern und Jugendlichen angesehen. Das Ziel besteht vor allem darin, dass unterbrochene oder blockierte Entwicklungsmöglichkeiten von allen Beteiligten wieder freigelegt und weiterverfolgt werden können. Die Eltern sollen befähigt werden, «die von Schuldgefühlen durchdrungene Angst in konstruktive Sorge und Anteilnahme umzuwandeln» (Novick \& Novick, 2005, dt. 2009, S. 79).

Im zweiten Kapitel Die Beziehung zwischen Eltern und Kind stellt Grieser die phasenspezifischen Entwicklungsaufgaben sowohl des Kindes bzw. Adoleszenten als auch der Eltern vor und geht besonders auf die Entwicklung der elterlichen 
Funktionen und die Fähigkeit der Eltern, sich als Team gegenseitig zu unterstützen ein. Wichtige Funktionen der Eltern für die Kinder liegen in der Befriedigung ihrer essenziellen Grundbedürfnisse wie Sicherheit, Versorgung, Geborgenheit, Liebe und Anerkennung, Orientierung und Gewissensbildung. Die besondere Bedeutung der Bindungs- und Empathie-Fähigkeit der Eltern und die «Passung» zwischen Eltern und Kind wird hervorgehoben. Auch das Kind erfüllt wichtige Funktionen für die Eltern, indem es für sie ein wichtiges narzisstisches Projekt ist, im günstigen Fall ihre positiven Erwartungen erfüllt und somit zu einer Quelle von Befriedigung und Kompetenzerleben wird. Im ungünstigen Fall kann das Kind aber auch zu einer Quelle von Enttäuschung, Neid, Hass, Scham- und Schuldgefühlen werden, zu Selbstwertproblemen der Eltern beitragen und unbewältigte Konflikte aus der Vergangenheit (die «Gespenster im Kinderzimmer») wieder aktualisieren.

Im Kapitel Diagnostik der Eltern-Kind-Beziehung führt Grieser aus, dass im Zentrum der Elternarbeit die Beziehung zwischen Eltern und Kind stehe (S. 27). Dies setzt bei der Therapeutin ein breites Spektrum an theoretischem Wissen und methodischen Konzepten voraus, um Verschränkungen der kindlichen Störung mit Auffälligkeiten der Eltern bzw. des Familiensystems psychodynamisch zu interpretieren und daraus Behandlungsziele sowie das geeignete Setting abzuleiten. Grieser empfiehlt, immer beide Eltern gemeinsam zum ersten Gespräch zu sehen und von diesem «Standardsetting» nur dann abzuweichen, wenn unüberwindliche Schwierigkeiten im Wege stehen (geografische Abwesenheit eines Elternteils, hochstrittige getrennte Eltern, Gewalt oder Drohungen). Alle anderen Gründe sind dahingehend zu prüfen, ob eine pathogene familiäre Dynamik inszeniert oder wiederholt wird und sich ein Widerstand gegen die Behandlung abbildet. Die Einführung des Settingelements «Elterngespräche finden immer mit beiden Eltern statt» könne daher bereits eine wichtige therapeutische Intervention darstellen (S. 28). Bei Patchworkfamilien, Heimkindern oder anderen Lebensformen werden andere wichtige Bezugspersonen $\mathrm{zu}$ «Elterngesprächen» eingeladen. Bei mangelnder Bereitschaft zur Mitarbeit eines Elternteils besteht die Gefahr, dass das Kind in einen Loyalitätskonflikt gerät und die Behandlung sehr wahrscheinlich scheitern wird. Die Befürchtungen der Eltern gegenüber einer Therapie des Kindes sind ernstzunehmen. Eltern haben mit Scham- und Schuldgefühlen zu kämpfen, können den Therapeuten als Zeuge des Scheiterns oder als potenziellen Aggressor empfinden oder können in Rivalität mit der Therapeutin geraten, die sie als kompetentere Person herbeisehnen und gleichermassen befürchten.

Bei der Diagnose der Eltern geht es um die Klärung der Frage, wie gut die Eltern ihre elterlichen Funktionen ausfüllen können, wie gut sie als Eltern-Team 
funktionieren, sich in der Partnerschaft unterstützt fühlen und welche Rolle, Funktion und Position dem Kind zugeschrieben wird (z.B. als narzisstisches Objekt, Partnerersatz, Störfaktor). Grieser geht auf verschiedene Dimensionen triadischer Kompetenz ein. Wie gut ist den Eltern die Erweiterung von der Paar-Dyade zur Eltern-Kind-Triade gelungen? Wie gut können sie das Kind «lesen», dessen Bedürfnisse mentalisieren, spiegeln und beantworten; mit Konflikten, Autonomieund Abhängigkeitsbedürfnissen umgehen; Grenzen setzen, Versagungen vertreten oder sich selbst schützen? Wichtige Einflussgrössen sind beispielsweise psychiatrische Störungen oder kompensierte Pathologien der Eltern, deren Strukturniveau, ihre Mentalisierungsfähigkeit, das Bewusstsein darüber, was den eigenen erzieherischen Stil geprägt hat, transgenerational bedeutsame Themen, Konflikte oder Traumata sowie familiäre Belastungen, Mythen und Tabus.

Im Zusammenhang mit dysfunktionalen Eltern-Kind-Beziehungen weist Grieser darauf hin, dass das Kind selbst durch konstitutionelle Faktoren oder Krankheiten seinen Teil zu einer schwierigen Eltern-Kind-Dynamik beiträgt und geht exemplarisch auf pathogene Beziehungsmuster ein. Das Verhalten des Kindes kann traumatische Vorerfahrungen der Eltern triggern, das Kind dadurch zum Auslöser von Distress und zum traumatischen Objekt werden. So kann das zunächst idealisierte Kind in eine Sündenbockrolle geraten.

In der Ablösungsdynamik der Adoleszenz geht es um die Problematik von elterlichen Delegationen, das Spannungsfeld von Bindungs- und Ausstossungstendenzen und die Verwicklung in Loyalitätskonflikte und Ablösungsschuld. Ein wichtiges Ziel der Elternarbeit besteht darin, diese Verknüpfungen aufzudecken und zu bearbeiten. Zur Frage, wie gut es den Eltern gelingt, mit ihrem Kind Beziehungen zu dritt zu gestalten, stellt Grieser vier Typen unvollständiger oder verzerrter Triaden dar (vgl. Grieser, 2015).

Er geht davon aus, dass Eltern alles ihnen Mögliche versucht haben, um das Kind zu fördern und die Probleme zu lösen. Eltern können nicht selten enttäuscht von sich sein, weil sie gescheitert sind und oft auch von ihrem Kind, weil dieses nicht so ist, wie sie es sich vorgestellt und gewünscht haben. Sie müssen lernen und akzeptieren, dass das reale Kind nicht so grossartig ist wie das «imaginäre Kind» und versuchen, sich mit dem realen Kind anzufreunden.

Im vierten Kapitel Rahmenvereinbarung und Klärung des Settings stellt Grieser verschiedene Settings dar: Elternarbeit alleine ohne Therapie des Kindes; Elternarbeit und Einzeltherapie des Kindes beim gleichen Therapeuten; Elternarbeit und Einzeltherapie des Kindes bei einem anderen Therapeuten; Elternarbeit in Elterngruppen; Familientherapie anstelle von Elternarbeit bei einer anderen 
Therapeutin. Er plädiert für eine ausführliche Abklärungsphase, bis ein tragfähiges Arbeitsbündnis mit den Eltern etabliert ist. In vielen Fällen sei es sinnvoll, «zunächst erst einmal nur mit den Eltern zu arbeiten und nur dann eine Einzeltherapie zu beginnen, wenn nach einem halben Jahr intensiver Elternarbeit noch immer Symptome bestehen» (S. 41). Wegen dem Abbruchrisiko ohne sorgfältige Abklärung warnt er davor, das Kind zu früh in Behandlung zu nehmen. Wenn sich das Kind der Einzeltherapie verweigert, kann andererseits auch eine Behandlung des Kindes über die Eltern praktiziert werden. Zudem kann durch eine sorgfältige und intensive vorbereitende Arbeit mit den Eltern eine bessere Grundlage für die Einzeltherapie geschaffen werden. Für klare Rahmenbedingungen ist auch eine genaue Klärung von Settingvariablen (Honorar, Frequenz, Dauer, Methodik, Schweigepflicht, Beendigung) wichtig. Spezifische Aspekte sind in der Arbeit mit getrennten, hochstrittigen, unvollständigen Elternpaaren und in der Elternarbeit bei Jugendlichen zu beachten.

Das abschliessende 5. Kapitel Elemente der Elternarbeit eröffnet mit der Bedeutung der therapeutischen Haltung. Die Therapeutin ist Hüterin des Settings und sorgt für die Einhaltung des Rahmens. In ihrem Bemühen um Empathie, eine haltende Beziehung und durch die Sicherheit des Rahmens erleben die Eltern beispielhaft eine Haltung, die für sie selbst im Umgang mit dem Kind wünschenswert wäre. Durch diese anerkennende Grundhaltung können sich Eltern entlastet fühlen, in dieser wohlwollenden Atmosphäre können neue Entwicklungskräfte freigelegt und alteVerhaltensmuster und Teufelskreise durchbrochen werden. Eine offene Haltung des Therapeuten gegenüber zunächst fremden und womöglich anderen Werten verpflichteten Eltern ist hilfreich, um die eigene Gegenübertragung handhaben zu können und keinen Abbruch von Seiten der Eltern zu provozieren. Das Bemühen um ein besseres Verstehen steht im Zentrum der therapeutischen Aufgabe. Weitere wichtige Fähigkeiten im therapeutischen Prozess sind: eine ausreichend gute triadische Kompetenz, die Fähigkeit Konflikte aufzudecken, die Abwehr der Eltern zu respektieren, das Aufdecken und Stärken von Ressourcen sowie die systemtherapeutischen Grundhaltungen der Neutralität und der Allparteilichkeit. Grieser empfiehlt, «mit der Offenheit und Neugier einer Ethnologin» auf die Eltern zuzugehen (S. 53), sich den Eltern aus einer Position des Nichtwissens anstatt aus einer des Experten zu nähern und sich ein gemeinsames Verständnis der konkreten Schwierigkeiten zu erarbeiten.

Neben den klassischen psychoanalytischen Techniken des Haltens, Klärens, der Arbeit mit Übertragung und Gegenübertragung, des Spiegelns, Verbalisierens, der Arbeit an Widerstand und Abwehr sowie der Deutung kommen auch das 
Vermitteln von Informationen zu entwicklungspsychologischen und psychodynamischen Zusammenhängen, von Kommunikationsregeln sowie psychoedukative Elemente zur Anwendung. Das Ratschläge-Geben wird problematisiert, weil diese nur zu oberflächlichen Veränderungen und Anpassungen führen und meistens auch nicht umgesetzt werden oder nicht funktionieren. Ziel der therapeutischen Arbeit sei ein vertieftes gemeinsames Verständnis der Problematik des Kindes, «weg vom Kreisen um die Symptome hin zum Blick auf das Kind in seinen Beziehungen» (S. 56). An die Stelle von Agieren soll Mentalisieren treten. Der Therapeut nutzt seine Gegenübertragungsgefühle als Spiegel dafür, was sich zwischen Eltern und Kind abspielt. Dazu skizziert der Autor exemplarisch Übertragungs-/ Gegenübertragungs-Konstellationen und Wege aus der Sackgasse. Der Abschnitt «Elternarbeit als Triangulierungsarbeit» zeigt auf, wie Triangulierungsprozesse angestossen und Eltern ihre elterlichen Kompetenzen und ihr Selbstwertgefühl entwickeln können. Eltern werden stets als Erwachsene und nicht als Patienten angesprochen, denn Kinder brauchen starke Eltern. Abschliessend umreisst der Autor, was in verschiedenen Phasen der Elternarbeit zu beachten ist.

Grieser gelingt es, im kompakten Format von 70 Seiten die Elternarbeit in der Psychotherapie von Kindern und Jugendlichen in ihren wesentlichen Aspekten $\mathrm{zu}$ beleuchten und in klarer Weise grundlegende Haltungen und Elemente der psychoanalytisch orientierten Arbeit dazustellen. Das Buch bietet eine gute Einführung in die Thematik, vor dem Hintergrund gut recherchierter Fachliteratur. So kann dieses übersichtliche, verständliche und klar strukturierte Buch sowohl AnfängerInnen auf diesem Gebiet wärmstens empfohlen werden als auch erfahreneren Fachpersonen, denen es einen guten Überblick und Anregungen in der Auseinandersetzung mit diesem anspruchsvollen Arbeitsfeld bietet.

\section{Literatur}

Datler, W., Figdor, H. \& Gstach, J. (1999). Die Wiederentdeckung der Freude am Kind. Psychoanalytisch-pädagogische Erziehungsberatung heute. Giessen: Psychosozial Verlag.

Grieser, J. (2015). Triangulierung. Giessen: Psychosozial-Verlag.

Novick, J. \& Novick, K.K. (2009). Elternarbeit in der Kinderpsychoanalyse. Klinik und Theorie. Frankfurt a.M.: Brandes \& Apsel. 

